\title{
EMIGRACIÓN, INMIGRACIÓN Y RETORNO: TRES ETAPAS DE UN MISMO PROCESO
}

\author{
DiegoLÓPEZ DE LERA \\ Universidad de La Coruña
}

Recibido: 03/03/2011

Aceptado: 30/06/2011

RESUMEN:El artículo aborda el tratamiento que en Europa se está dando al retorno de los trabajadores extranjeros extracomunitarios a sus países de origen, tomando como ejemplo el caso de España, uno de los países que han recibido más inmigrantes en este joven siglo XXI, el primero dentro de la región europea.

Se presenta una breve síntesis de las principales teorías sobre el retorno migratorio, una tipología ideal de "migrantes retornados" y la situación actual del conocimiento que tenemos sobre las corrientes de retorno tanto en Europa como en el resto de las principales áreas de recepción migratoria.

Se describe la situación del "retorno" en España dos años después de empezar la crisis financiera mundial de 2007, afectada por un grave paro laboral. Se analizan los datos estadísticos oficiales por regiones de origen-destino y las políticas y programas específicos que se han desarrollado en España en torno al retorno de inmigrantes extranjeros, tanto las ayudas a personas en situación de vulnerabilidad como las ayudas a los inmigrantes que tienen reconocido pago por prestación de desempleo, dentro del marco de la política europea sobre retorno.

PALABRAS CLAVE: Europa, migración de retorno, España, retorno según origen-destino, políticas específicas.

\section{EMIGRATION, IMMIGRATION AND RETURN, THREE STAGES OF THE SAME}

PROCESS

ABSTRACT: The article discusses the treatment in Europe is taking the return of foreign workers from outside their countries of origin, taking the example of Spain, one of the countries that more immigrants have received in this young 21 st century, the first in European region.

One presents a brief synthesis of the main theories of return migration, an ideal typology of "returning migrants" and the current state of knowledge we have about the currents of return, both in Europe and in the rest of the principal areas of receipt of migrantes.

It describes the situation of "return" in Spain two years after beginning the global financial crisis of 2007 , affected by a serious labor unemployment. There is analyzed the official statistical data by regions of origin-destination and the policies and specific programs that have developed in Spain concerning the return of foreign immigrants, both the aid to persons in vulnerable situation such as the aid to immigrants who have recognized unemployment benefit, inside the frame of the European politics on return.

KEY WORDS: Europe, returning migration, Spain, regions of origin-destination, specific policies. 


\section{INTRODUCCIÓN}

El tema de estudio que se presenta a continuación es el retorno migratorio, en concreto el tratamiento que en Europa se está dando al retorno de los trabajadores extranjeros extracomunitarios a sus países de origen, tomando como ejemplo el caso de España, afectada por un grave paro laboral, a raíz de la crisis financiera de 2007 y económica de 2008 (sector de la construcción, automotriz, etc).

Dado que el retorno puede ser considerado como parte de la contracorriente de inmigración, su magnitud y características están relacionadas, por lo que empezaremos con un breve resumen de lo que ha sido la inmigración en España en lo que va de siglo XXI. Como es conocido dentro del contexto europeo, el aumento de la inmigración que ha recibido España en lo que va de siglo ha sido de una intensidad inesperada. Razón que en algunos sectores generó la idea de que la crisis financiera internacional que estalló en 2007 podía ocasionar un retorno generalizado entre la población inmigrante, cuyo volumen sería considerable a la vista del número de posibles afectados. Sin embargo, a la vista de la experiencia a dos años vista del comienzo de la crisis, parece que la idea ha resultado exagerada.

Algunos rasgos que perfilan la inmigración recibida son los siguientes. Durante el primer lustro de este siglo (2000-04) el promedio de entradas alcanzó la cifra de los 600000 inmigrantes anuales, la intensidad de la migración continuo creciendo y en 2007 se alcanzó la cifra máxima con casi 900000 inmigrantes. En términos cuantitativos se trata de cantidades que se podrían considerar como "desproporcionadas" para un país como España, si se compara con la magnitud del resto de variables demográficas de una población de casi 47 millones de personas ${ }^{1}$ (ver GRÁFICO 1). El crecimiento exponencial de la inmigración entre los años 2000 y 2008 hizo que se incorporaran cerca de 4 millones y medio de personas, lo que supuso que el aporte migratorio pasara a predominar ampliamente el crecimiento demográfico, llegando a suponer más de tres cuartas partes del crecimiento total de la población del país ${ }^{2}$ (ver GRÁFICO 2).

Esta inmigración ha colocado a España entre los países que han recibido más inmigrantes en este joven siglo XXI, el primero dentro de la región eu-

\footnotetext{
${ }^{1}$ En términos comparativos, el aporte total de la natalidad no alcanzaba los 450000 nacimientos anuales, en la misma época.

2 Estamos hablando del aporte migratorio "directo", sin incluir su aporte al crecimiento vegetativo a través de la nupcialidad y la natalidad.
} 
ropea. De esta forma, España se unió con ímpetu aunque tarde, al grupo de países europeos de inmigración.

Poco a poco las sociedades receptoras de Europa Occidental, incluidas la española y la portuguesa, han ido asumiendo que el destino de la mayoría de los inmigrantes es el de quedarse, formar una familia y mezclarse con los autóctonos. En la medida en que estas sociedades han ido tomando conciencia de este proceso, se ha ido dando importancia a la integración social y se han ido poniendo en marcha políticas específicas al respecto ${ }^{3}$.

Gráfico 1. España. Tasas brutas de los componentes de la dinámica demográfi$c a$

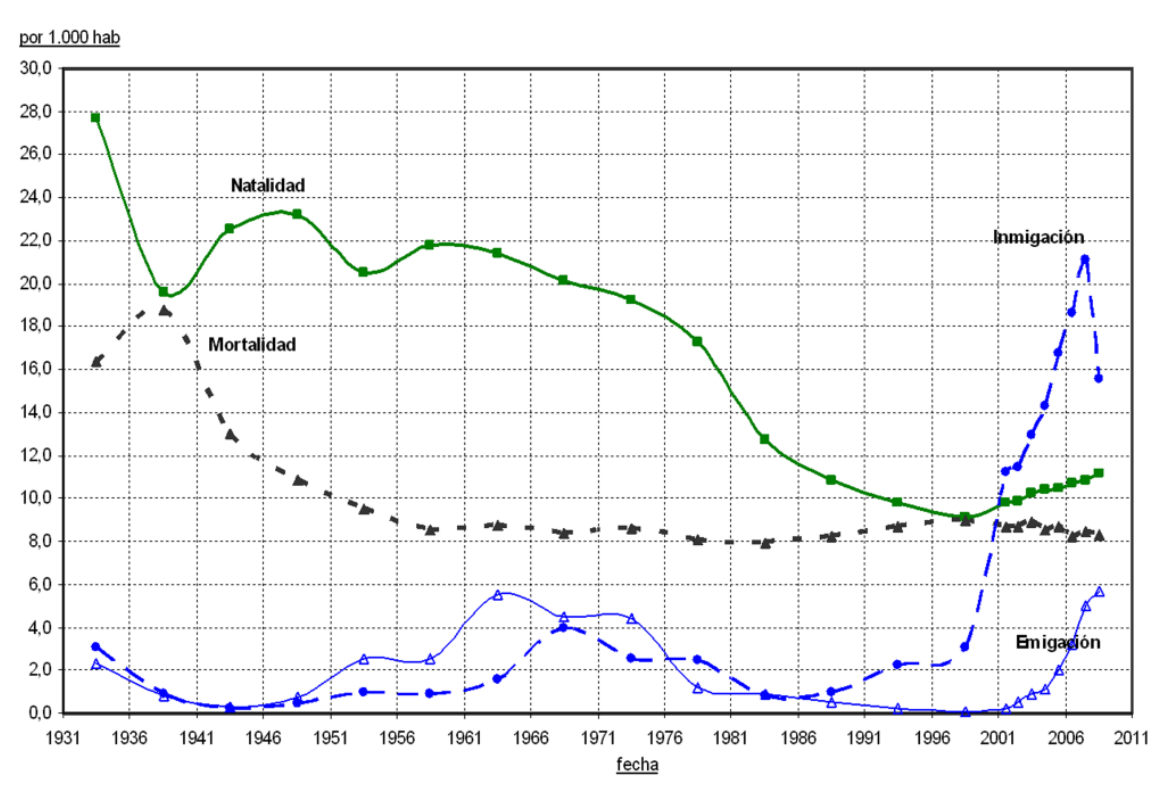

FUENTE: ESOMI. Elaboración propia. Corrección de datos sobre emigración española. Estimación inmigración de extranjeros. INE, Movimiento Natural de la Población.

${ }^{3}$ Un buen ejemplo lo tenemos en España con la creación en 2005 del "Fondo de apoyo a la acogida e integración de inmigrantes y el refuerzo educativo". El Fondo distribuye entre las CCAA un crédito presupuestario (entre 150 y 200 millones de euros), para actuaciones en materia de integración de inmigrantes y de refuerzo educativo, que se formaliza a través de convenios de colaboración entre el Ministerio de Trabajo y Asuntos Sociales y las Comunidades Autónomas. El organismo coordinador es el Consejo Superior de Política de Integración. 
Gráfico 2. España. Tasas brutas del crecimiento demográfico

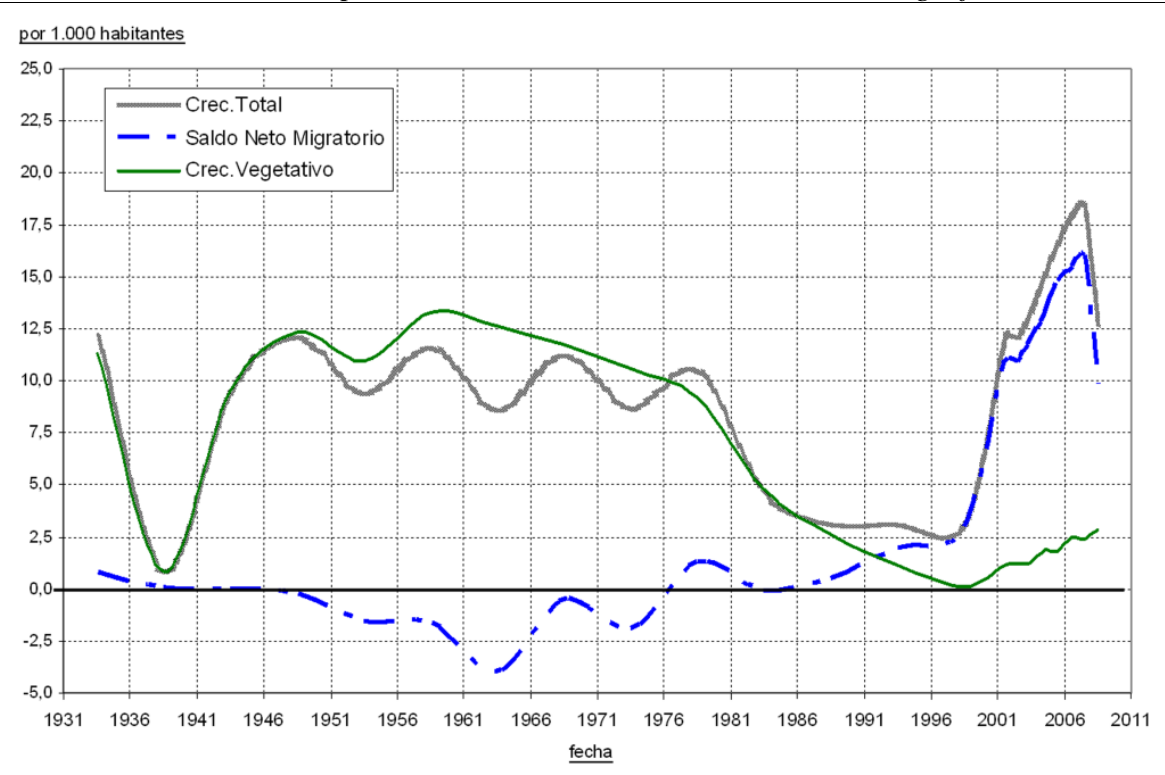

FUENTE: ESOMI. Elaboración propia. Corrección de datos sobre emigración española. Estimación inmigración de extranjeros. INE, Movimiento Natural de la Población.

Con todo esto se ha ido difuminando la idea oficial de "temporalidad" asociada a la presencia de población inmigrada, que atraviesa la política de la Unión Europea en materia de inmigración, pero, sin embargo, la idea del retorno sigue estando presente en la suposición de que muchos inmigrantes acabarán volviéndose a su país de origen.

De hecho, la intensa corriente inmigratoria recibida ha tenido su consecuente contracorriente de retorno, cuyo volumen ha sido mucho menos intenso (ver GRÁFICO 3), pero que desde 2008, como se indicó, ha cobrado interés a raíz de la crisis económica mundial, que en España ha afectado drásticamente el mercado laboral.

Según las estadísticas sobre migraciones internacionales, el retorno es una contracorriente inseparable en toda corriente migratoria. Desde el punto de vista de los países de origen, se habla por lo general de un desfase de dos a cinco años entre las corrientes de emigración y retorno. La intensidad del retorno depende de la intensidad de la primera; del tiempo transcurrido y de cambios que modifiquen el desequilibrio socioeconómico de partida entre origen y destino.

Así como las sociedades europeas han ido asumiendo la "permanencia" de los inmigrantes, éstos, por su parte, también van acostumbrándose a la idea 
de "quedarse" a medida que van sorteando dificultades y ahondando sus raíces (consiguen su permiso de residencia, reagrupan a su familia o forman una nueva, compran una casa para dejar de "gastar" en alquiler y así aumentar su ahorro, escolarizan a sus hijos).

Gráfico 3. España. Migración internacional

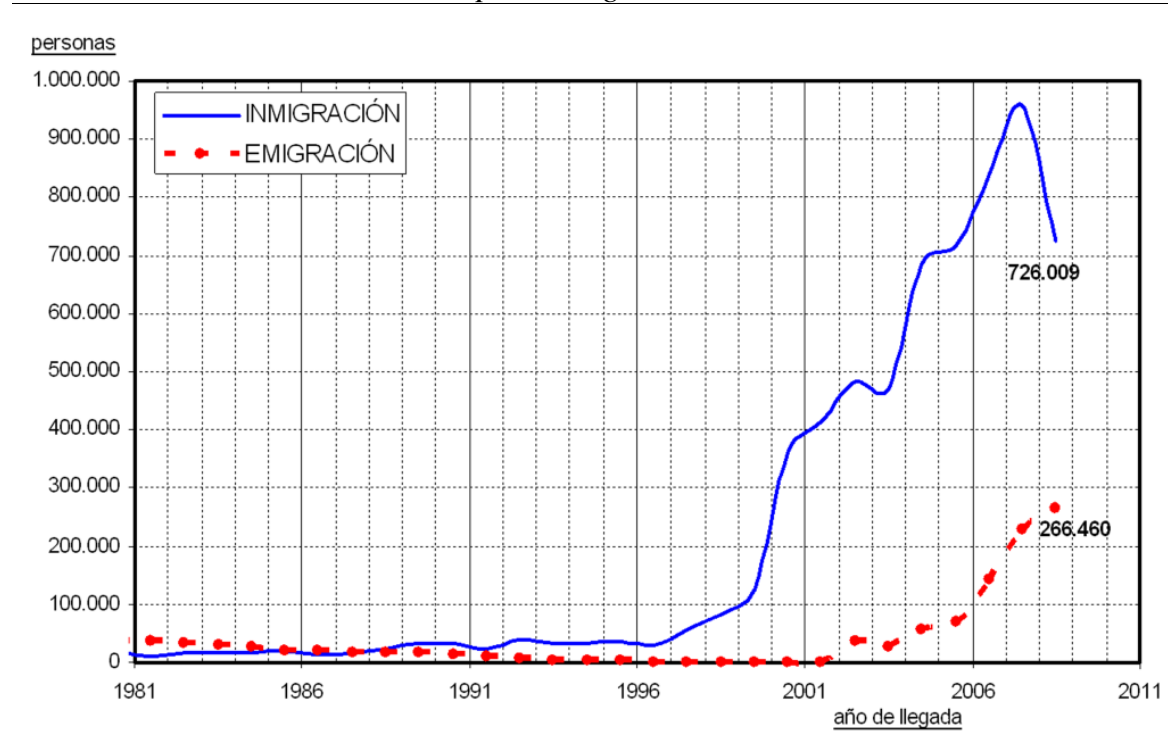

FUENTE: ESOMI. Elaboración propia. INE, Estadística de Variaciones Residenciales NotA: Los datos de emigrantes corresponden en un $87 \%$ a inmigrantes extranjeros que se van de España y en un 3\% a inmigrantes españoles nacidos en el extranjero ( $2^{\text {as }}$ generaciones) que retornan después de haber probado suerte en España (\% de 2008).

Por lo general, la mayoría de los inmigrantes responden afirmativamente cuando se les pregunta si piensan volver a su país de origen ${ }^{4}$, la idea del retorno va con elloscuando se van de su país, pero esa aspiración se va alterando con el paso del tiempo. La percepción de bienestar es algo relativo, depende de la comparación con la situación anterior y también de la valoración de las posibilidades de futuro (aquí y allí). Cuando el discurso se va acercando al momento actual, alejándose de los planes de partida, el discurso se tor-

${ }^{4}$ En algunas corrientes migratorias (Argentina, Cuba, Venezuela) hay emigrantes que tienen claro desde el principio que no quieren volver. En muchos casos, son personas cualificadas que contaban con trabajo y una situación económica estable, pero que buscan mejorar su "calidad de vida", es decir vivir en un ambiente con menos violencia, mas seguridad (social, jurídica y política), donde las normas se cumplan. 
na más concreto ${ }^{5}$. En la medida en que se van consiguiendo metas y la situación laboral va mejorando se valoran las posibilidades de "consolidar" la posición alcanzada. Así como también se compara con las posibilidades que se van a encontrar a la vuelta, tanto para los migrantes como para sus hijos. Cuando estos últimos han nacido aquí sus proyectos no suelen coincidir con los de los padres, la idea del "retorno" supone para ellos un desarraigo que no aceptan de buen grado. Comprenden que sus padres hubieran pasado por eso, cuando salieron de su país y vinieron aquí, pero sus padres lo habían elegido así y exigen que a ellos también se les deje elegir.

En el actual contexto de crisis internacional, a todos estos factores se les suma el hecho de que la situación en el país de origen sigue tan mala como estaba cuando se fueron, sino peor. De forma que la decisión de volver se va postergando (aunque no desaparezca de su discurso), poco a poco se va haciendo menos probable, aunque en el futuro siga siendo posible.

\section{ESTADO DE LA CUESTIÓN}

\section{I.1. Bases teóricas}

Lo general es emigrar pensando en volver. Las metas de los emigrantes suelen ser temporales y no definitivas, y sus períodos relativamente cortos. Muchos emigrantes comprueban como poco a poco la realidad va disolviendo el sueño del retorno definitivo, y los nuevos lazos personales y compromisos adquiridos son los que les obligan a echar raíces en otras tierras.

El retorno no es un proceso automático, una consecuencia directa de una variable externa, el logro de una meta parcial; un cambio de régimen; la caída de un dictador, implica un proceso complejo de toma de decisiones y de evaluaciones personales y familiares, tanto en el lugar de acogida como en el lugar e origen.

Hay un componente de "género" en la decisión del retorno, pues es común la opinión que los hombres tienden al retorno y las mujeres tienden al establecimiento. $\mathrm{Y}$ a la hora de realizar el balance entre costes y beneficios las mujeres migrantes tienen mucho más que perder que ganar.

La teoría neoclásica afirma que la decisión de migrar es una determinación racional, hecha de acuerdo a un cálculo de costes y beneficios (BORJAS,

\footnotetext{
${ }^{5}$ Se trata de entrevistas en profundidad realizadas a finales de 2009 en Valencia y Madrid a inmigrantes latinoamericanos, marroquíes y rumanos. Dentro del marco de un estudio sobre "Procesos de retorno de los inmigrantes extranjeros en España", Universidad de Coruña (MICINN. CSO2008-03561).
} 
1989). La decisión de retorno es similar, pero la perspectiva, el momento, el cúmulo de información y la situación del migrante son distintos. En el retorno se puede realizar un cálculo de costos y beneficios con conocimiento de causa directa: se sabe lo que es trabajar y vivir en el extranjero y se es consciente del costo personal de quedarse de manera definitiva.

Lo anterior tiene que ver con la reflexión sobre la lógica del emigrante de "ganar en euros y gastar en pesos" (como por ejemplo los ecuatorianos, peruanos o colombianos en España o los mexicanos en EEUU), pero este argumento carece de sentido cuando se queda a vivir en España, pues se gana un salario mínimo y sus gastos son en euros. El migrante puede tener movilidad social en su país de origen si regresa, pero en el lugar de destino la mayoría de los inmigrantes estarán ubicados en los niveles más bajos de la escala social.

A diferencia del pasado (siglo XIX y primeras décadas del siglo XX), hoy en día las posibilidades del migrante de mejorar su nivel de vida en los países occidentales de destino son muy limitadas, pues son absorbidos por un mercado de trabajo segmentado que los relega a los puestos de trabajo que paulatinamente van abandonando los trabajadores autóctonos (PIORE, 1979). Los inmigrantes se ubican en el mercado secundario donde es fácil llegar a un tope salarial y es difícil salir de ese círculo vicioso. A pesar de la oportunidad de los enclaves étnicos, una posible opción de movilidad social se da en un contexto de retorno, y este argumento es definitivo en un cálculo ampliado de costes y beneficios.

En el momento de la partida, el migrante optará por el retorno o la permanencia como meta, estas decisiones primarias marcan los esfuerzos y sacrificios de la vida cotidiana del trabajador migrante y orientan el destino de las remesas. Los migrantes que piensan en el retorno mantienen sus vínculos con el lugar de origen, cuidan de sus relaciones e incrementan su capital social a lo largo del tiempo (MASSEY, ALARCÓN, DURAND, GONZÁLEZ, 1987); (MASSEY, 1993); (MASSEY, 1996). El capital social puede servir tanto para emprender la aventura migratoria como para el retorno. En este sentido, la teoría del capital social explica la factibilidad del retorno.

\section{I.2. Tipos de retornados}

Siguiendo a otros estudios, la versatilidad del retorno se puede resumir en al menos cinco tipos (DURAND, 2004); (GUALDA, 2004); (MASSEY, 1987); (SCHRAMM, 2009):

Retorno final. Migrante que regresa de manera definitiva y voluntaria después de una larga estancia: jubilados, los que retornan en edades in- 
termedias con la mejora de las oportunidades económicas del país, retorno de exiliados políticos, etc. Se trata del retorno voluntario de aquellos que vuelven después de muchos años y no tienen ni obligación ni necesidad de ello, pues están instalados en el lugar de destino, tienen la documentación en regla, e incluso, propiedades y familia.

$\checkmark$ Retorno temporal. Retorno de trabajadores temporales sujetos a programas específicos donde el contrato exige u obliga al retorno (Programa Bracero entre México y Estados Unidos, 1942-64).

$\checkmark$ Retorno transgeneracional. La migración de retorno transgeneracional, es decir, no del migrante sino de su descendencia: hijos, nietos, bisnietos, etc., donde se aducen lazos sanguíneos y culturales para facilitar el ingreso o la naturalización. Es ocasiones este tipo de retorno es fomentado políticamente por los países de origen (el caso español e italiano).

$\checkmark$ Retorno forzado. Casos de retornos de pueblos enteros por razones políticas o raciales (deportación masiva de mexicanos en la década de los 20 y 30 en EEUU), y las deportaciones a raíz de las políticas restrictivas de entrada en países del primer mundo. A este particular conviene mencionar la reciente normativa aprobaba en la Unión Europea sobre Retorno (Directiva de retorno, junio de 2008, Parlamento Europeo), que aumenta las posibilidades de "repatriar" a inmigrantes.

$\checkmark$ Retorno fracasado. El retorno voluntario de los fracasados después de una experiencia negativa, cuyas causas y razones deben analizarse a través de técnicas etnográficas.

\section{LO QUE SABEMOS DEL RETORNO EN LA ACTUALIDAD}

\section{II.1. La medición internacional del retorno}

Reconocida esta situación, no es menos cierto que siempre ha existido una importante corriente de retorno en todo proceso migratorio, el hecho de que no retornen muchos no quiere decir que no lo hagan unos cuantos. Las corrientes de "retorno" han crecido proporcionalmente a medida que la mejora de los medios de transporte han ido "acercando" (en tiempo, dinero y acceso) a los migrantes con los lugares de destino y origen. En Europa, actualmente las proporciones de retornados pueden situarse alrededor del 50\% (Irlanda, Bélgica, Reino Unido) o del 25\% (Holanda), en Estados Unidos se estima que ronda el 19\% (OCDE, 2008: 171). Sin embargo la "medición" del retorno continúa planteando serios problemas analíticos, tanto de naturaleza (definición de "retornado"), como de medición (registro de salidas V.S. vuelta al lugar de origen), compatibilidad (uso de distintas fuentes estadísticas) y de cobertura.

En otras palabras, si la medición de las migraciones no resulta fácil y es, 
en comparación con el resto de las variables demográficas, la de menor cobertura en su cuantificación, en el caso concreto de las migraciones de retorno nos encontramos ante una lamentable pobreza estadística (PAJARES, 2009: 167).

Sabemos por algunos estudios recientes que aproximadamente y dependiendo del lugar de destino entre un 20 y un $50 \%$ de los inmigrantes abandonan el país en los cinco primeros años de su llegada, sea para volver a su país de origen o para migrar a otro destino (migraciones secundarias). Así como que ciertos países, como Canadá, los Estados Unidos o Nueva Zelanda tienen mayor éxito que los países europeos a la hora de retener a sus inmigrantes (OCDE, 2008: 163). Aquí conviene especificar que se están sumando los "retornos" al país de origen y las "segundas migraciones" a nuevos destinos. Es decir se puede estimar cuántos inmigrantes salen del país de destino, pero no se sabe a dónde se han dirigido.

En los casos que se ha podido estimar este porcentaje de retorno "real" (inmigrantes que vuelven a su país de origen) se ha constatado que varía sensiblemente, según el país de destino y los lugares de origen (OCDE, 2008: 174). Por ejemplo, en España se estima que han regresado (en los cinco años de su llegada) el $16 \%$ de los chilenos frente al 4,3\% de los argentinos. Si lo comparamos con EEUU el retorno de los chilenos apenas llega a la mitad (7\%), aunque el de los argentinos es muy similar $(3,8 \%)$.

En cifras globales, se puede afirmar que entre los inmigrantes de las sociedades de Europa occidental hay mayor propensión al retorno (entre un $40 \%$ y $60 \%$ ) que entre los inmigrantes de Estados Unidos, Canadá o Nueva Zelanda (alrededor de 20\%)

Nos estamos refiriendo exclusivamente a procesos de retorno que implican un periodo de residencia más o menos prolongado en el país de destino (más de un año) antes de producirse el retorno. Quedan fuera algunos tipo de migraciones en los que la ida y vuelta (emigración y retorno) forman parte consustancial del proyecto migratorio (migraciones circulares; fronterizas, estacionales).

\section{II.2. Situación de la emigración en España}

En el caso de España, en los siete años (2002-08) para los que se tiene datos estadísticos sobre la emigración al extranjero se han registrado casi 660.000 salidas de extranjeros ${ }^{6}$, lo que supone el $17 \%$ de la población ex-

\footnotetext{
${ }^{6}$ La Estadística de Variaciones Residenciales (EVR) publicó por primera vez datos sobre
} 
tranjera residente en España a mitad de período.

Desde 2002 la progresión de la emigración ha sido claramente creciente, como se ha podido observar en el GRÁFICO n. ${ }^{\circ} 3$, y con un desplazamiento de 6-7 años con respecto a la serie de inmigración. Es de esperar que los datos para 2009 confirmen esta tendencia.

Estadísticamente hablando la serie no es homogénea, pues junta las "bajas de extranjeros" con las "bajas por caducidad". Las primeras son voluntarias, lo que las hace sufrir de un subregistro, aún no estimado, pero que no debe ser despreciable. Las segundas corresponden a la puesta en práctica en $2005^{7}$ de la Ley orgánica 14/2003 promulgada dos años antes, que obligaba a los extranjeros extracomunitarios que no tuvieran permiso de residencia permanente a "actualizar" su alta padronal cada dos años, en caso contrario las autoridades locales tienen autorización para darlos de baja del padrón municipal, por eso los primero datos corresponden a 2005.

La intención de las "bajas por caducidad" es disminuir el subregistro de las "bajas de extranjeros" que por su carácter voluntario son difíciles de corregir, por lo que al sumarlas ${ }^{8}$ se puede tener una mejor idea de la magnitud de la emigración de extranjeros que se está produciendo en España ${ }^{9}$.

Si dejamos fuera las "bajas por caducidad" (61\% del total en 2008 , ver GRÁFICO 4), nos quedamos con el 39\% que representan las "bajas de extranjeros", de las cuales solo se conoce el destino del $42 \%$ de ellas, según los datos de $2008 \%$ (ver GRÁFICO 5).

$\mathrm{Si}$ analizamos este $42 \%$, se observa que la principal corriente de retorno está formada por europeos (21\%) y la segunda por latinoamericanos (16\%), a lo lejos le siguen las corrientes de africanos $(4 \%)$ y asiáticos $(1,5 \%)$.

Sin embargo, es conveniente matizar que en el conjunto de emigrantes que se van a Europa se pueden distinguir dos grupos socialmente diferentes, por un lado están los que se dirigen a los 15 países de la antigua Unión Europea más Suiza, que suponen una migración de ocio residencial, compuesta ma-

bajas al extranjero en 2002. Antes de esa fecha existía otra estadística, la de "Emigración asistida de españoles", pero que, como indica su propio nombre, solo cubría la salida de españoles que se acogían a las ayudas oficiales a la emigración.

${ }^{7}$ BOE del 30-05-2005.

${ }^{8}$ La equivalencia de las "bajas por caducidad" con las "bajas al extranjero" es responsabilidad del autor.

${ }^{9}$ Obviamente no toda la emigración de extranjeros es retorno, aunque sirve como aproximación, ya que solamente un pequeño porcentaje se va a un país distinto del país de origen. 
Gráfico 4. Bajas de extranjeros al extranjero

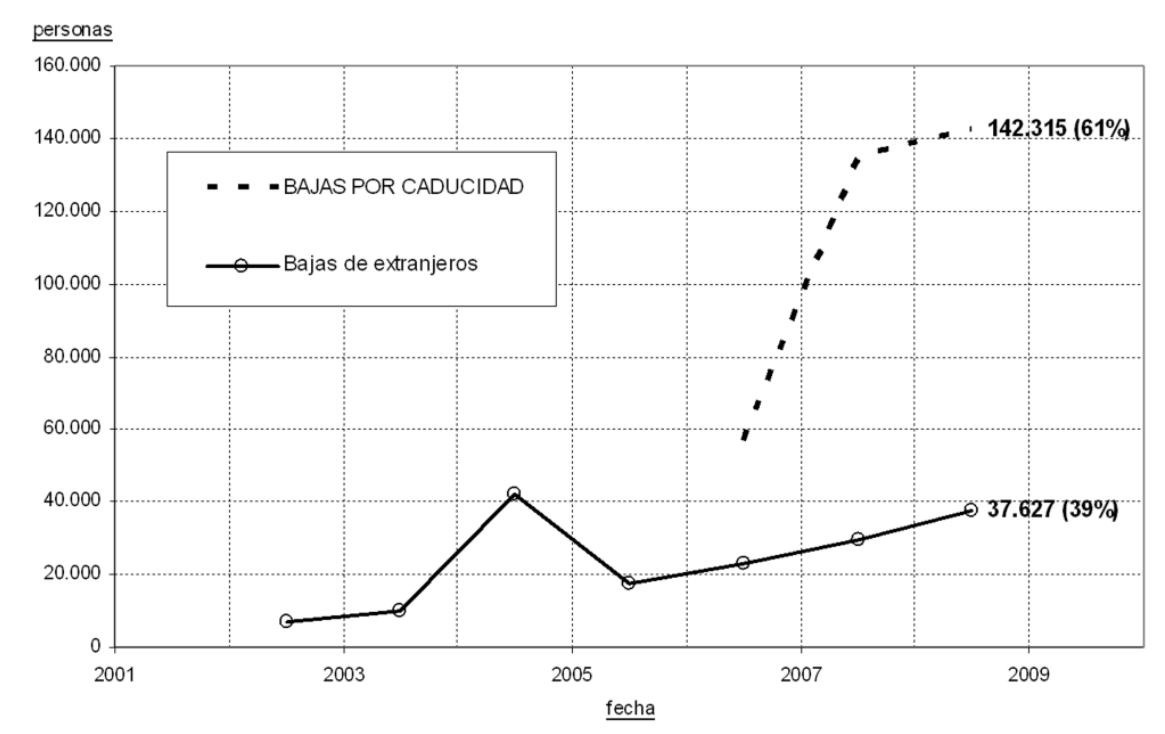

FUENTE: ESOMI. Elaboración propia. INE, Estadística de Variaciones Residenciales.

Gráfico 5. España. Emigrantes extranjeros al extranjero, según región de destino

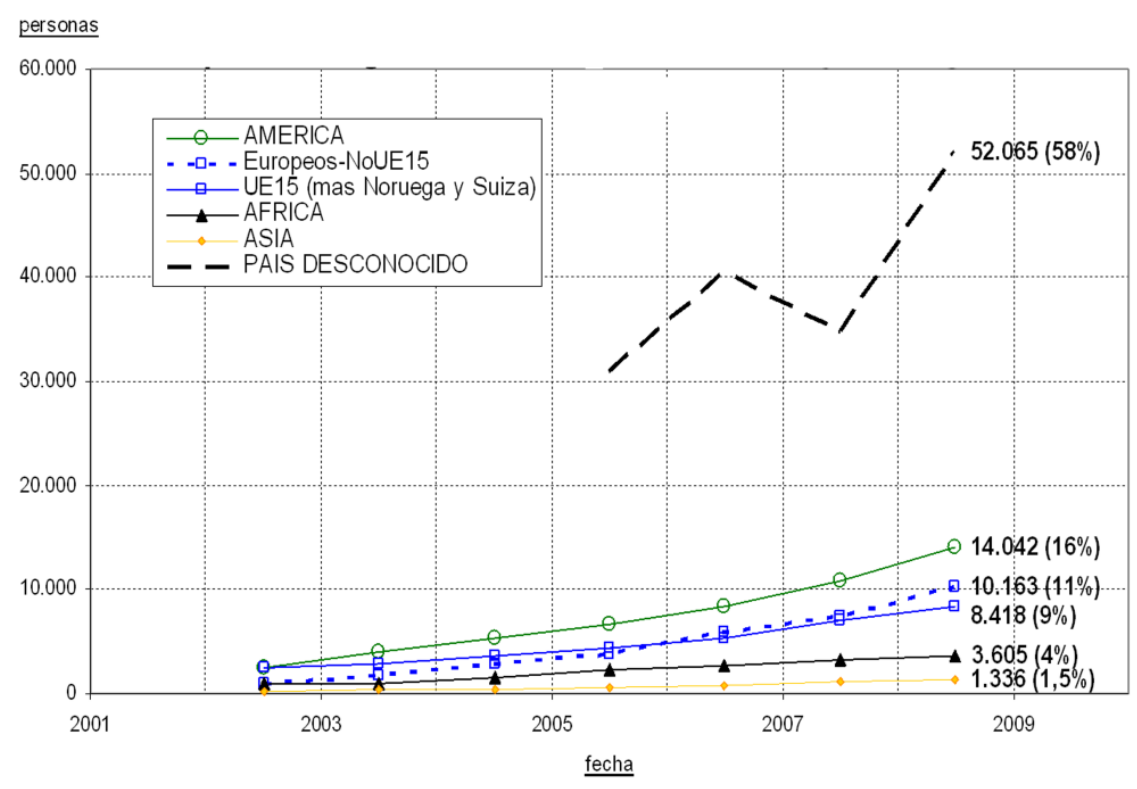

FUENTE: ESOMI. Elaboración propia. INE, Est. de Variaciones Residenciales. 
yoritariamente por personas mayores jubiladas. Por otro están los que se dirigen al resto de países europeos, entre los que predominan trabajadores de países del este europeo (ver GRÁFICO 6). Se trata de una corriente migratoria de carácter laboral comparable al resto de las series.

Si eliminamos la emigración hacia la Europa de los 15, los latinoamericanos (Bolivia, Brasil, Argentina, Colombia, Ecuador) pasan a dominar claramente la emigración laboral de extranjeros, seguidos por los europeos del este, entre los que destacan los rumanos, que son la corriente emigratoria más numerosa incluso antes de la crisis económica.

Gráfico 6. España. Emigrantes extranjeros al extranjero, según país de destino (sólo bajas con país conocido - $16 \%$ del total)

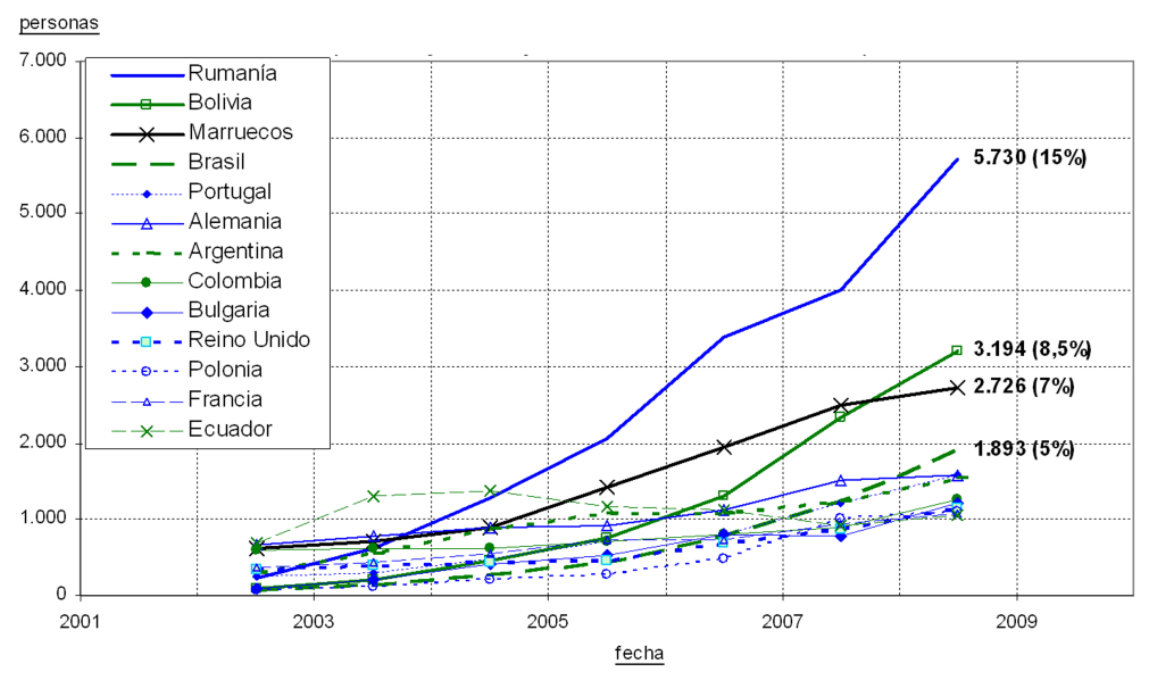

FUENTE: ESOMI. Elaboración propia. INE, Est. de Variaciones Residenciales.

\section{POLÍTICAS DE RETORNO}

\section{III.1. Europa}

Desde 2000 hasta 2007 ha operado en la Unión Europea el Fondo Europeo para los Refugiados. Su objetivo general del FER ha sido el de apoyar los esfuerzos de los Estados miembros en la mejora de la gestión de todas las dimensiones del retorno a través del uso del concepto de gestión integral y la promoción de acciones conjuntas a ser implementadas por los Estados miembros. El fondo apoya también a las acciones nacionales que cumplen con los principios de solidaridad comunitarios. En términos de Retorno Voluntario, el FER apoya la implementación justa y efectiva de los estándares comunes del retorno ente los Estados miembros, e incluye medidas relacio- 
nadas con el retorno voluntario de personas que no tienen la obligación legal de abandonar el territorio.

Estas medias ejecutadas bajo el Fondo Europeo para los Refugiados durante los anteriores periodos de programación (2000-2007), a partir del año 2008 forman parte del Fondo Europeo para el Retorno, aprobado por el Parlamento Europeo (decisión 575/2007) y el Consejo europeo para el período 2008-2013, como parte del Programa general Solidaridad y Gestión de los Flujos Migratorios, que está dotado de 676 millones de euros (UNIÓN EUROPEA, 2007). El objetivo general del nuevo FER pretende gestionar, de manera integrada, el retorno de la población inmigrante reforzando la cooperación entre los Estados miembros y fomentando la aplicación de normas comunes.

Esta política convive con otras iniciativas, como la reciente normativa aprobaba en la Unión Europea sobre Retorno. La aprobación en junio de 2008 por el Parlamento Europeo de la Directiva de Retorno (denominada también Directiva de la infamia o Directiva de expulsión) consolida el proceso de involución que sobre los derechos humanos se viene produciendo en la Unión Europea cuando se trata de legislar sobre inmigración desde que el miedo a la inmigración irregular se incardinó en sus instituciones. Si se leen las directivas europeas sobre inmigración se comprueba claramente que el "control de fronteras" ha sido la piedra angular de la política migratoria comunitaria.

Si bien las legislaciones de extranjería de los años ochenta contenían normas que regulaban el internamiento y la expulsión no es hasta la Directiva 2001/40/CE que comienza a tomar forma una política comunitaria centrada en la inmigración irregular y las expulsiones de migrantes (AGUELO); (CHUECA, 2008). Desde entonces y hasta la actualidad las medidas de retorno son "una piedra angular de la política de migración de la UE" (COMISIÓN EUROPEA, 2007).

\section{III.2. España}

Actualmente España cuenta con dos programas de retorno asistido que se gestionan con fondos públicos. Uno que funciona desde 2003, en el que intervienen diversas organizaciones, pero que fue impulsado inicialmente por la Organización Internacional para las Migraciones (OIM) a través de un convenio con el Ministerio de Trabajo e Inmigración; y otro puesto en marcha en 2008, coincidiendo con la crisis económica, que facilita el retorno de trabajadores extranjeros en paro con derecho a prestación por desempleo. 
Estas medidas cuentan con el apoyo de fondos europeos a través del Fondo Europeo para el Retorno, que ascienden a $25255838,81 €$ para el período 2008-13. El Ministerio de Trabajo e Inmigración es el responsable de la implementación de las acciones nacionales de retorno voluntario en España durante este periodo de programación, mientras el Ministerio del Interior es el responsable de los otros aspectos de la gestión del Fondo. Cada ministerio ejecutará las actuaciones según la estrategia de programación plurianual (2008-2013) que establece el uso al que se destinarán los fondos recibidos de forma anual

a. Programa de retorno voluntario para inmigrantes en situación de vulnerabilidad (PREVIE)

Como se ha dicho el programa denominado PREVIE (Programa de Retorno Voluntario de Inmigrantes desde España), está gestionado por la OIM y se puso en marcha en julio de 2003, a través de un convenio entre el Ministerio de Trabajo y la OIM ${ }^{10}$.

Por parte del Estado la gestión del programa esta coordinada por la " $\mathrm{Di}$ rección General de Integración de los Inmigrantes" ", aunque tiene un desarrollo específico en las comunidades de Cataluña y de Madrid.

Hasta 2008 este programa se gestionó a través de nueve organizaciones: ACCEM (Asociación Comisión Católica Española de Migraciones); ACOBE (Asociación de Cooperación Bolivia-España); AESCO (Asociación España-Colombia); CARITAS; CEPAIM; Cruz Roja; FEDROM (Federación de asociaciones de emigrantes rumanos en España); MPDL (Movimiento por la Paz); RESCATE.

Los inmigrantes a los que va dirigido ese programa son personas que han de llevar en España más de seis meses y se encuentran en situación de vulnerabilidad social, lo que debe probarse por medio de la presentación de un informe de los servicios sociales del ayuntamiento en el que residen (o en su defecto de una ONG especializada). La voluntariedad del retorno, por otra parte, debe ser manifiesta, por lo que se les exige la firma de un impreso de voluntariedad.

Por medio de este programa ${ }^{12,}$ a los inmigrantes se les aporta información y orientación sobre el retorno, ayuda en la tramitación de la documentación

\footnotetext{
${ }^{10}$ España se incorporó como miembro de la OIM en 2005.

${ }^{11}$ Dependiente de la Secretaria de Estado de Inmigración y Emigración del Ministerio de Trabajo e Inmigración (MTIN).
} 
necesaria para el retorno, billetes de viaje, una pequeña cantidad de dinero de bolsillo para el viaje, la posibilidad de ayuda económica para la reintegración en el país de origen y, finalmente, la posibilidad de seguimiento sobre su reinserción en el país de origen. En el plano económico esto se ha concretado en el pago del billete, ayuda económica de viaje y la aportación de 400 euros de ayuda por persona, hasta un máximo de 1600 euros por familia para gastos de reinstalación en su país.

El número de personas retornadas por medio de este programa es muy inferior al de las personas que regresan "por su cuenta". Por lo general, se duda de que la existencia de programas de retorno asistido suponga incrementos significativos sobre el retorno general que se produce. Sin embargo, el retorno asistido es valorado positivamente por las organizaciones internacionales, en los casos de personas en situación de vulnerabilidad.

En España el número de inmigrantes acogidos a este programa desde 2003 hasta marzo de 2009 asciende a las 6671 personas (que representa aproximadamente $1 \%$ de las bajas de la EVR entre 2003 y 2008). El perfil corresponde en su gran mayoría a inmigrantes en situación irregular; personas que han visto fracasar sus intentos de conseguir trabajo y por lo tanto de conseguir "papeles". La mayoría son de origen latinoamericano y con hijos en sus países de origen.

La finalidad del programa no es, por lo tanto, la de reducir el número actual de desempleados, sino su capacidad para resolver situaciones concretas de vulnerabilidad.

b. Programa de retorno voluntario con capitalización de las prestaciones por desempleo (APRE)

Este programa surge a finales de 2008 (MINISTERIO, 2008b), cuando ya se percibe un fuerte incremento del paro entre los inmigrantes. Se trata de un programa "ad hoc" creado a raíz de la crisis laboral generada por las repercusiones de la crisis financiera de 2007 y con la intención de "incentivar" el retorno de trabajadores extranjeros

Los requisitos para acogerse a este programa son los siguientes (www.planderetornovoluntario.es):

\footnotetext{
${ }^{12}$ Para mas información se puede consultar la página web de la OIM www.iomemadrid.es/ index.php/programas-proyectos/retrono-voluntario\#vulnerabilidad.
} 
Ser nacionales de alguno de los 20 países extracomunitarios que tienen suscrito con España un convenio bilateral en materia de Seguridad Soci$\mathrm{al}^{13}$.

$\checkmark$ Estar inscritos como demandantes de empleo en el Servicio Público de Empleo correspondiente.

Encontrarse en situación legal de desempleo como consecuencia de la extinción de la relación laboral.

$\checkmark$ Tener reconocido el derecho a la prestación por desempleo del nivel contributivo, sin compatibilizarlo con un trabajo a tiempo parcial.

$\checkmark$ Comprometerse a:

$\checkmark$ Retornar al país de origen en el plazo máximo de 30 días naturales contados desde la fecha del primer pago de la prestación.

$\checkmark$ Hacerlo, en su caso, en compañía de los familiares reagrupados sin una autorización de residencia independiente.

$\checkmark$ No retornar a España en el plazo de 3 años para residir y/o realizar una actividad lucrativa o profesional.

Se trata de unos requisitos que limitan el acceso a los extranjeros no comunitarios que disponen de permiso de residencia y de trabajo y que tiene derecho a percibir una cobertura por desempleo, es decir que hayan cotizado lo suficiente para percibir esta prestación.

A pesar de estas limitaciones, pueden acogerse a este programa la mayoría de los principales colectivos de inmigrantes que residen en el país. Sin embargo quedan excluidos algunas importantes corrientes de inmigrantes como la de rumanos (14\% del total de extranjeros a principios de 2008) y la de bolivianos (5\%), así como prácticamente todos los países africanos (exceptuando Marruecos y Túnez, 5\%) y asiáticos (a excepción de Filipinas, 4\%).

El abono de la prestación por desempleo se realiza en dos plazos: el $40 \%$ en España, una vez reconocido el derecho, y el $60 \%$ en el país de origen, entre los 30 y 90 días naturales desde la fecha del primer pago de la prestación. Para el $2^{\circ}$ cobro es necesario presentarse personalmente en la representación diplomática u oficina consular española en el país de origen.

El Ministerio de Trabajo e Inmigración, a través de la Secretaría de Estado de Inmigración y Emigración, puede complementar el abono anticipado de la prestación contributiva por desempleo con ayudas para el viaje a sus países de origen. Estas ayudas incluyen el pago del billete internacional desde Es-

13 Andorra; Chile; Filipinas; República Dominicana; Argentina; Colombia; Marruecos; Túnez; Australia; Ecuador; México; Ucrania; Brasil; Estados Unidos; Paraguay; Uruguay; Canadá; Federación Rusa; Perú y Venezuela. 
paña a su país; en caso necesario, el abono de los gastos de desplazamiento en España desde su domicilio actual hasta la ciudad de salida a su país de origen, pudiendo incluir el abono de los gastos de alojamiento de una noche por motivos de viaje; la concesión de una ayuda económica de viaje de 50 euros por cada uno de los miembros de la unidad familiar. Así como el pago de gastos imprevistos debidamente justificados.

El compromiso de esperar tres años para poder volver a solicitar una nueva autorización de residencia o trabajo implica de hecho la pérdida del derecho de residencia para todos aquellos que se acojan al programa. Esto afecta también a los familiares dependientes, de modo que quienes hubiesen sido reagrupados por el solicitante y aún no tuviesen una autorización de residencia independiente, perderán también su derecho de residencia. Según los testimonios recogidos, la pérdida de la autorización de residencia supone un obstáculo muy serio para la mayoría de los inmigrantes, les ha costado muchos meses, trabajo y penalidades reunir las condiciones necesarios para obtener este permiso y renunciar al mismo se les hace cuesta arriba, aunque estén sin trabajo.

Por éste y otros motivos la respuesta que está teniendo el Programa ha quedado lejos de satisfacer las previsiones iniciales. Desde octubre de 2008 hasta junio de 2009 han solicitado esta ayuda alrededor de 5000 personas ( $2 \%$ de las bajas de la EVR en 2008), de las cuales se han denegado menos del $10 \%$ y el resto está en tramitación y/o en espera a que haya fondos disponibles.

Según los testimonios recabados, hay muchas personas que desean retornar pero que no cubren los requisitos, por lo que están volviendo por su cuenta o en algunos casos acudiendo a las jefaturas de policía para ser "expulsados" del país por carecer de permiso de residencia en vigor, lo que conlleva a su repatriación.

Por el contrario hay otros que reúnen todos los requisitos pero se ven obligados a retrasar su vuelta porque no saben que hacer con su "piso". A medida que fueron progresando, adoptaron la costumbre española de comprar casa y pagar hipoteca en vez de alquiler, como forma de ahorro. Pero se encuentran actualmente con una sobreoferta de viviendas en venta debido a la crisis inmobiliaria que les hace imposible vender su casa o renegociar la hipoteca y no encuentran como deshacerse de la hipoteca que han contraído con el banco para irse de España y volver a su país.

Otros muchos siguen sopesando la oportunidad de volver. La situación en su país sigue tan mal como cuando se fueron y no tienen claro que salgan 
ganando retornando allí. Sobre todo si para ello tienen que perder su "permiso de residencia".

Como se indicó, la nacionalidad es una de las limitaciones para acogerse al programa de retorno voluntario de 2008, pero no solamente porque quedan excluidos un buen número de países, sino también porque quedan descartadas las familias de inmigrantes en las que hay miembros que han adquirido la nacionalidad española. Esto afecta principalmente a los inmigrantes latinoamericanos, que son los que suelen nacionalizarse como mayor frecuencia, dadas las facilidades que tienen para adquirir la nacionalidad frente a los otros grupos de extranjeros extracomunitarios.

\section{BIBLIOGRAFÍA}

Aguelo, P.; Chueca Sancho A. G. (2008):«Directiva de retorno, directiva de expulsión: (nota crítica avuelapluma desde los derechos humanos)». Revista de derecho migratorio y extranjería, n. $^{\circ} 18,121-166$.

BORJAS, G. J. (1989): «Economic theory and internacional migration». International Migration Review, vol. XXIII, number 3, 457-485.

CABALLERO GuALDA, E. (2004): «El retorno de los españoles: una nueva emigración», en CHECA, F.; CHECA, J.C.; ARJONA, A. (edits.), Inmigración y Derechos Humanos. La integración como participación social. Barcelona, Icaria.

Comisión EuRopea (2005): Comunicación de la Comisión. Programa de La Haya: Diez prioridades para los próximos cinco años. Una asociación para la renovación europea en el ámbito de la libertad, la seguridad y la justicia. Bruselas, Comisión Europea.

COMISIÓN EUROPEA (2007): Comunicación de la Comisión. Tercer Informe anual sobre inmigración e integración. Bruselas, Comisión Europea.

Devolder, D.; GIl Alonso, F.; ForTe, P. Bas (2006): «Estimación del grado de error en el registro de la población extranjera en España: un enfoque comparativo», en X Congreso de la Población Española. Migraciones, movilidad y territorio. Pamplona.

DURAND, J. (2004): «Ensayo teórico sobre la migración de retorno. El principio del rendimiento decreciente». Cuadernos Geográficos, $\mathrm{n}^{\circ}$ 35, 103-116

GARCÍA, P.; QUICIOS GARCÍA, M. (2010):«La otra cara de la inmigración: educación para el retorno». Revista Iberoamericana de Educación, n. ${ }^{\circ}$ 51/3.

HERRERA, G. (2004): «Elementos para una comprensión de las familias transnacionales», en HIDALGO, Francisco (ed.) - Migraciones. Un juego con cartas marcadas. Quito, Ed. Abya-Yala, 215- 232.

LÓPEZ DE LERA, D. (2006): «Panorama de la inmigración», en EsCRIBANO, A. (ed.), Demografía de los extranjeros. Incidencia en el crecimiento de la población. $\mathrm{La}$ Coruña: Ed. Fundación BBVA, pp.17-72.

LÓPEZ DE LERA, D. (2006): «El impacto de la inmigración extranjera en las regiones españolas», en Análisis territorial de la demografía española (2006). Madrid, Fundación Fernando Abril Martorell. 
Massey, D. S.; Alarcón, R.; Durand, J.; GonZÁlez, H. (1987): Return to Aztlán: The Social Process of International Migration from Western Mexico. Berkeley: University of California Press.

MASSEY, D. S.; ARANGO, J. et ALIA (1993): «Theories of International Migration: A Review and Appraisal». Population and Development Review, vo-lume 19, number 3, 431-466.

Massey, D. S.; Arango, J.; Hugo, G.; Kouaoooucu, A.; Pellegrino, A.; TAYLOR, E. (1996):Worlds in Motion. Understanding International Migration at the End of the Millenium. Oxford, Clarendon Prees.

MEJíA, W. (2008): «El retorno como dimensión clave de la migración internacional para las zonas de origen, el caso de Pereira y su área metropolitana (AMCO)», en Primer Seminario Internacional Dimensiones para una Política Pública Distrital Migratoria.

MejíA, W.; Toro, G., (2003): «Migraciones y micrográfico: el caso del Eje Cafetero colombiano». Cultura y Droga, año 8, n. $^{\circ} 10$.

Ministerio De LA PRESIDENCIA (2008 ${ }^{\mathrm{a}}$ : Boletín Oficial del Estado, n. ${ }^{\mathrm{o}}$ 228, de 20 de septiembre de 2008. Real Decreto Ley 4/2008 de 19 de septiembre, sobre abono acumulado y de forma anticipada de la prestación contributiva por desempleo a trabajadores extranjeros no comunitarios que retornen voluntariamente a sus países de origen.

Ministerio DE LA PRESIDENCIA (2008b): Boletín Oficial del Estado, n. ${ }^{\circ}$ 272, de 11 de noviembre de 2008. Real Decreto 1800/2008, de 3 de noviembre, por el que se desarrolla el Real Decreto-ley 4/2008, de 19 de septiembre, sobre abono acumulado y de forma anticipada de la prestación contributiva por desempleo a trabajadores extranjeros no comunitarios que retornen voluntariamente a sus países de origen.

OCDE (2008): «Return migration: a new perspective», enInternational migration outlook: SOPEMI-2008 Edition.

PAJARES, M. (2009): Inmigración y mercado de trabajo. Informe 2009. Madrid, Observatorio Permanente de la Inmigración (OPI).

PINO, M. R.; VERDE, C. (2006): «Emigración de retorno: análisis de la situación a través de historias de vida». Migraciones, n. $^{\circ}$ 20, 201-230.

PIORE, M. J. (1979): Birds of Passage. Migrant, Labor in Industrial Societies. Cambridge, Cambridge University Press.

PRIES, L. (ed.) (1997): «Transnationale Migration». Soziale Welt, Sonderband 12. Baden-Baden, Nomos.

SCHRAMM, C. (2009): «La importancia de redes sociales transnacionales para el retorno y la reinserción en el proceso migratorio de migrantes ecuatorianos», en Ponencia presentada al VI Congreso sobre Inmigración en España.

Unión EuROPEA (2007): Diario Oficial de la Unión Europea, L144 6.6.2007, Decisión no 575/2007 del Parlamento Europeo y del Consejo de 23 de mayo de 2007 por la que establece el Fondo Europeo para el Retorno para el período 2008-2013 como parte del Programa general "Solidaridad y Gestión de los Flujos Migratorios". 